\author{
N. Sakhraoui, A. Chefrour \& S. Metallaoui
}

\title{
Naturalisation de Melia azedarach (Meliaceae) et premier signalement de Canna indica (Cannaceae) et Pelargonium zonale (Geraniaceae) en Algérie
}

\begin{abstract}
Sakhraoui, N., Chefrour, A. \& Metallaoui, S.: Naturalisation de Melia azedarach (Meliaceae) et premier signalement de Canna indica (Cannaceae) et Pelargonium zonale (Geraniaceae) en Algérie. - Fl. Medit. 29: 223-226. 2019. — ISSN: 1120-4052 printed, 2240-4538 online.

In this contribution, we report the naturalization of Melia azedarach and the discovery of Canna indica and Pelargonium zonale growing outside the usual cultivation sites, making this work the first report of these species in Algeria. Some species must be monitored because they are invasive in the Mediterranean area.
\end{abstract}

Key words: alien flora, xenophytes, mode of reproduction, invasive character, Algeria.

\section{Introduction}

Bien qu'elles participent à l'enrichissement de la flore nationale, plusieurs espèces de la flore naturalisée, qui ne cesse d'augmenter en Algérie, sont considérées comme envahissantes ou potentiellement envahissantes (Meddour \& El Mokni 2016) et peuvent de ce fait constituer un véritable danger pour la biodiversité locale et les écosystèmes en général (Shine \& al. 2000).

Nous rapportons ici, la découverte de trois espèces exotiques proliférant en dehors des lieux de cultures habituels, faisant de ce travail, le premier signalement de ces espèces en Algérie. Les plantes en question, dont certaines possèdent un caractère envahissant, ont été observées lors de la réalisation de prospections floristiques dans la wilaya de Skikda (Sakhraoui \& al. 2019). Cette étude met le point sur certaines de leurs caractéristiques biologiques et écologiques permettant de mieux comprendre leur comportement dans notre pays, rendant ainsi possible la prévention contre d'éventuelles futures invasions.

\section{Matériel et Méthodes}

L'identification des espèces a été effectuée grâce à la consultation de Maire (1959), Maas-Van de Kamer \& Maas (2008) et Cullen \& al. (2011). La présentation des familles végétales suit l'Angiosperme Phylogeny Group (APG III 2009). La nature des 
milieux colonisés et le mode de reproduction ont été déterminés in situ. Le type biologique a été donné selon Raunkiaer (1934), quant au degré de naturalisation, il a été évalué selon Magnanon \& al. (2008).

\section{Résultats et discussion}

\section{Melia azedarach L. (Meliaceae)}

Phanérophyte, originaire de l'Asie du sud (Yulianti \& al. 2011), introduite par le jardin d'essai du Hamma avant 1843 (Payen 1845). Observée durant cinq années consécutives de 2014 à 2019 çà et là dans les ruelles de la cité Larbi Ben M'hidi et le long de la route reliant la cité à la ville de Skikda, où elle se présente souvent en pieds isolés poussant parmi la flore indigène. Les fruits ont été observés mais la germination des graines n'a pas été enregistrée. Les dimensions considérables des individus observés indiquent qu'ils se sont établis dans la région depuis plus de dix années ce qui nous permet de les considérer comme complètement naturalisés.

\section{Canna indica L. [ $\equiv$ C. aurantiaca Roscoe, $\equiv$ C. cearensis Huber] (Cannaceae)}

Géophyte, originaire de l'Amérique tropicale (Maire 1959), introduite en 1838 par le jardin d'essai du Hamma (Carra \& Gueit 1952). Observée à plusieurs points de la cité Salah Chebel et à l'endroit appelé « El guelta » (commune de Hamadi Krouma) où de petites populations, séparées les unes des autres, prolifèrent parmi les espèces indigènes. Elle a aussi été retrouvée au bord de la route reliant Stora à la ville de Skikda. Les fruits ont été observés, mais la germination des graines n'a pas été enregistrée. En voie de naturalisation, car son apparition dans la région est récente (moins des 10 années requises).

\section{Pelargonium zonale (L.) L'Hér. [三 Geranium zonale L.] (Geraniaceae)}

Phanérophyte, originaire d'Afrique du Sud (Cullen \& al. 2011), probablement introduite par le jardin d'essai du Hamma, Battandier \& al. (1914) rapportent que $P$. zonale était naturalisée dans le jardin botanique d'Alger. Retrouvée en 2017 dans plusieurs points de la cité Salah Chebel (commune de Hamadi Krouma) et en 2019, au bord de la route reliant Filfilla à Hamrouche Hamoudi, et dans une décharge à la cité Larbi Ben M'Hidi. Les fruits ont été observés mais la germination des graines n'a pas été enregistrée. Subspontanée, car la plante ne se propage pas dans le milieu pour se mêler à la flore indigène.

Toutes les espèces sont considérées comme naturalisées dans d'autres régions du monde (Henderson 2007; Uludağ \& al. 2017; Domina \& al. 2018; Domingues De Almeida 2018; Galasso \&al. 2018), où elles ont été introduites comme plantes d'ornement. En Afrique du Nord, C. indica est signalée naturalisée en Tunisie (El Mokni 2018), alors que P. zonale est signalée cultivée/parfois subspontanée à Madère et à statut problématique aux Canaries et au Maroc (Dobignard \& Chatelain 2012).

En Algérie, à l'exception du M. azedarach, rapportée par Dobignard \& Chatelain (2012) cultivée/parfois subspontanée dont nous signalons la naturalisation complète pour la première fois dans le pays, les deux autres espèces n'ont pas été signalées auparavant dans les ouvrages traitant la flore algérienne notamment Quezel \& Santa (1962; 1963) et Dobignard \& Chatelain (2010-2013). 
$C$. indica et $M$. azedarach sont considérées comme envahissantes notamment dans le bassin méditerranéen (Capdevilla Argüelles \& al. 2006; Arianoutsou \& al. 2010; Podda \& al. 2011). Bien qu'elles ne soient pas encore considérées comme invasives avérées dans notre région, elles pourraient le devenir dans les prochaines années, favorisées par le changement climatique que connait l'Algérie et l'intensification des perturbations des milieux naturels et semi naturels dans la wilaya de Skikda générée par les projets de développement local. En effet, le réchauffement climatique et les perturbations des milieux sont considérés comme des facteurs susceptibles d'amplifier les invasions biologiques (Gritti \& al. 2006; Walther \& al. 2009).

Ces espèces devraient donc figurer sur une liste de surveillance et être soumises à des contrôles périodiques permettant la détection précoce de toute invasion réelle.

\section{Références}

APG III. 2009: An update of the Angiosperm Phylogeny Group. Classification for the orders and families of flowering plants. APG III. - Bot. J. Linn. Soc. 161: 105-121. https://doi.org/10.1111/j.1095-8339.2009.00996.x

Arianoutsou, M., Bazos, I., Delipetrou, P. \& Kokkoris, Y. 2010: The alien flora of Greece: Taxonomy, life traits and habitat preferences. - Biol. Invasions 12: 3525-3549. https://doi.org/10.1007/s10530-010-9749-0

Battandier, J.A., Maire, R. \& Trabut, L.C. 1914: Rapport sur les herborisations faites par la Société pendant la session d'Alger. - Bull. Soc. Bot. Fr. 61(5): 325https://doi.org/10.1080/00378941.1914.10832611

Capdevilla Argüelles, L., Iglesias Garcia, A., Orueta, J.F. \& Zilleti, B. 2006: Especies exoticas invasoras: Dignostico y bases para la prevencion y el manejo. Série Técnica. - Madrid.

Carra, P. \& Gueit, M. 1952: Le jardin d'essai du Hamma. Gouvernement général de l'Algérie, Direction de l'Agriculture. - Alger.

Cullen, J., Knees, S. G. \& Cubey, H.S. 2011: The European garden flora flowering plants, Resedaceae to Cyrillaceae. $2^{\text {nd }}$ ed., 3. - Cambridge.

Dobignard, A. \& Chatelain, C. 2010-2013: Index synonymique de la flore de l'Afrique du Nord, 1-5. - Genève.

Domina, G., Galasso, G., Bartolucci, F. \& Guarino, R. 2018: Ellenberg Indicator Values for the vascular flora alien to Italy. - Fl. Medit. 28: 53-61. https://doi: 10.7320/FlMedit28.053.1

Domingues De Almeida, J. 2018: New additions to the exotic vascular flora of continental Portugal. - Fl. Med. 28: 259-278. https://doi: 10.7320/FlMedit28.259

El Mokni, R. 2018: Canna indica L. (Cannaceae). Pp 199-200 in: Raab-Straube E. von \& Raus Th. (eds), Euro+Med-Checklist Notulae 9. $\quad-\quad$ Willdenowia 48: 195-220. https://doi.org/10.3372/wi.48.48203

Galasso, G, Conti, F., Peruzzi, L., Ardenghi, N. M. G., Banfi, E., Celesti-Grapow, L., Albano, A., Alessandrini, A., Bacchetta, G., Ballelli, S., Bandini Mazzanti, M., Barberis, G., Bernardo, L., Blasi, C., Bouvet, D., Bovio, M., Cecchi, L., Del Guacchio, E., Domina, G., Fascetti, S., Gallo, L., Gubellini, L., Guiggi, A., Iamonico, D., Iberite, M., Jiménez-Mejías, P., Lattanzi, E., Marchetti, D., Martinetto, E., Masin, R.R., Medagli, P., Passalacqua, N. G., Peccenini, S., Pennei, R., Pierini, B., Podda, L., Poldini, L., Prosser, F., Raimondo, F. M., Roma-Marzio, F., Rosati, L., Santangelo, A., Scoppola, A., Scortegagna, S., Selvaggi, A., Selvi, F., Soldano, A., Stinca, A., Wagensommer, R. P., Wilhalm, T. \& Bartolucci, F. 2018: An updated checklist of the vascular flora alien to Italy. - Pl. Biosyst. 152: 556-592. http://doi.org/10.1080/11263504.2018.1441197 
Gritti, E. S., Smith, B. \& Sykes, M. T. 2006: Vulnerability of Mediterranean Basin ecosystems to climate change and invasion by exotic plant species. - J. Biogeogr. 33: 145-157. https://doi.org/10.1111/j.1365-2699.2005.01377.x

Henderson, L. 2007: Invasive, naturalized and casual alien plants in southern Africa: a summary based on the Southern African Plant Invaders Atlas (SAPIA). - Bothalia 37(2): 215-248.

Maas-Van de Kamer, H. \& Maas, P.J.M. 2008: The Cannaceae of the world. - Blumea 53: 247-318. http://dx.doi.org/10.3767/000651908X607945

Magnanon, S., Geslin, J., Lacroix, P. \& Zambettakis, C. 2008: Examen du statut d'indigénat et du caractère invasif des plantes vasculaires de basse Normandie, Bretagne et pays de la Loire. Proposition d'une première liste de plantes invasives et potentiellement invasives pour ces régions. - Bull. Conserv. Bot. Natl. Brest E.R.I.C.A. 21: 73-104.

Maire, R. 1959: Flore de l'Afrique du Nord, 6. - Paris.

Meddour, R. \& El Mokni, R. 2016: État de l'art sur les plantes envahissantes ou à caractère invasif introduites en Algérie et en Tunisie. XV OPTIMA Meeting, 6-11 June 2016. - Montpellier.

Payen, M. 1845: Rapport sur les travaux de M. Hardy, directeur de la pépinière centrale du gouvernement, à Alger. - Monit. Algérien 658: 1-4.

Podda, L., Fraga i Arguimbau, P., Mascia, F., Mayoral Garcia-Berlanga, O. \& Bacchetta, G. 2011: Comparison of the invasive alien flora in continental islands: Sardinia (Italy) and Balearic Islands (Spain). - Rend. Fis. Acc. Lincei. 22(1): 31-45. https://doi: 10.1007/s12210-010-0112-2

Quézel, P. \& Santa, S. 1962-1963: Nouvelle flore d'Algérie et des régions désertiques méridionales, 1-2. - Paris.

Raunkiaer, C. 1934: The life forms of plants and statistical plant, Geography. - Oxford.

Sakhraoui, N., Metallaoui, S., Chefrour, A. \& Hadef, A. 2019 : La flore exotique potentiellement envahissante d'Algérie: première description des espèces cultivées en pépinières et dans les jardins. - Biotechnol. Agron. Soc. Environ. 23(2): 63-73. https://doi.org/10.25518/17804507.17902

Shine, C., William, N. \& Gunbdling, L. 2000: A guide to designing legal and institutional frameworks on alien-invasive species. Droit et politique de l'environnement, 40. Gland. - UICN.

Uludağ, A., Aksoy, N., Yazlık, A., Filiz Arslan, Z., Yazmış, E., Üremiş, I., Cossu, T. A., Groom, Q., Perg, J., Pyšek, P. \& Brundu, G. 2017: Alien flora of Turkey: checklist, taxonomic composition and ecological attributes. - NeoBiota 35: 61-85. https://doi: 10.3897/neobiota.35.12460

Walther, G. R., Roques, A., Hulme, P. E., Sykes, M. T., Pysek, P., Kuhn, I. \& Zobel, M. 2009: Alien species in a warmer world: risks and opportunities. -Trends Ecol. Evol. 24(12): 686-693. https://doi: 10.1016/j.tree.2009.06.008

Yulianti, Y., Siregar, I. Z., Wijayanto, N., Tapa Darma, I. \& Syamsuwida, D. 2011: Genetic variation of Melia azedarach in community forests of West Java assessed by RAPD. - Biodiversitas 12(2): 64-69. https://doi: 10.13057/biodiv/d120202

Adresses des auteurs:

Nora Sakhraoui $^{1 *}$, Azzedine Chefrour ${ }^{2} \&$ Sophia Metallaoui ${ }^{1}$,

${ }^{1 *}$ Département des Sciences de la Nature et de la Vie (SNV). Faculté des sciences. Université 20 Aout 1955, BP 26 Route d‘El-Hadaiek, Skikda, 21000, Algérie. Email: sakhraouinora05@gmail.com; metallaoui_s@yahoo.fr

${ }^{2}$ Département de Biologie. Faculté des Sciences de la Nature et de la Vie. Université Mohamed Cherif Messaadia, Souk Ahras, 41000, Algérie. E-mail: azchefrour@yahoou.fr

* auteur correspondant 